\title{
Bacteriological Profiles and Antibiotic Susceptibility Patterns in Complicated Bacterial Infections of the Ears, Nose and Throat
}

\author{
FLORIN ANGHELINA ${ }^{1}$, OVIDIU ZLATIAN ${ }^{2}$, MIRCEA SORIN CIOLOFAN ${ }^{1}$, DANIELA CALINA ${ }^{3}$, \\ ANCA OANA DOCEA ${ }^{4}$, RAZVAN HAINAROSIE ${ }^{5}$, OCTAVIAN DRAGOS PALADE ${ }^{6}$, \\ LILIANA ANGHELINA ${ }^{7}$, CRISTIAN DRAGOS STEFANESCU ${ }^{5}$, LAURA MAZILU ${ }^{8}$, \\ ANDRA IULIA SUCEVEANU ${ }^{8}$, CATALINA PIETROSANU ${ }^{5}$, ANDREI OSMAN ${ }^{9}$ \\ ${ }^{1}$ University of Medicine and Pharmacy, Otorhinolaryngology Discipline, 2nd Petru Rares Str., 200349, Craiova, \\ Romania \\ ${ }^{2}$ University of Medicine and Pharmacy, Microbiology, Virology, Parasitology Discipline, 2nd Petru Rares Str., 200349, \\ Craiova, Romania \\ ${ }^{3}$ University of Medicine and Pharmacy, Department of Clinical Pharmacy, 2 Petru Rares Str., 200349, Craiova, Romania \\ ${ }^{4}$ University of Medicine and Pharmacy, Department of Toxicology, 2 Petru Rares Str., 200349, Craiova, Romania \\ ${ }^{5}$ Carol Davila University of Medicine and Pharmacy, 8th Eroii Sanitari Blvd., 050474, Bucharest, Romania \\ ${ }^{6}$ Grigore T. Popa University of Medicine and Pharmacy,Otorhinolaryngology Discipline, Second Surgery Department, \\ 16th Universitatii Str., 700115, Iasi, Romania \\ ${ }^{7}$ University of Medicine and Pharmacy, Paediatric Discipline, 2nd Petru Rares Str., 200349, Craiova, Romania \\ ${ }^{8}$ Ovidius University, Faculty of Medicine, 124 Mamaia Blvd., 900527, Constanta, Romania \\ ${ }^{9}$ University of Medicine and Pharmacy, Anatomy Discipline, 2nd Petru Rares Str., 200349, Craiova, Romania
}

\begin{abstract}
The objective of this study was to identify and map bacteriological profiles and antibiotic susceptibility patterns of complicated bacterial infections in the otorhinolaryngological (ENT) spectrum. Antibiotic resistance was another goal of our study. We defined complicated bacterial infections as: acute bacterial pathology of the ears, nose and throat with complications that required immediate surgical approach, chronic diseases of the ears, nose and throat that required surgical treatment due to ongoing bacterial infections, middle ear cholesteatoma with complications, complicated suppurated tonsillitis in children, intratonsillar abscess, parapharyngeal space abscess, cellulitis of the neck, face and/or involving cervical ganglia and neoplasms of the pharynx or larynx with an acute ongoing bacterial superinfection. The study was carried out over a period of 12 months, on a total number of 82 patients (children and adults), admitted in our department for the complicated bacterial diseases listed above. For the ENT spectrum, we uncovered that more than 50\% of complicated bacterial infections were caused by 4 pathogens: Pseudomonas species, Staphylococcus aureus non-MRSA and MRSA species and Streptococcus species (other than Beta-haemolytic Group A Streptococci and Streptococcus pneumoniae). Almost two thirds of all complicated bacterial infections are complicated cases of sinusitis or mastoiditis that require immediate surgical treatment due to possible life-threatening complications.
\end{abstract}

Keywords:complicated bacterial infection, bacteriological profiles, antibiotic resistance, bacterial culture, bacterial antibiotic susceptibility.

Upper respiratory tract infections (URTI) are extremely frequent illnesses caused by an acute infectious process which involves the upper respiratory tract. In some cases, when certain more complicated URTIs lack proper medical treatment, they may evolve into chronic diseases or may develop complications. Bacterial infections cause more complications and require antibiotic treatment, therefore a lot of studies have focused on describing bacterial profiles for organ-related infections, and the susceptibility of bacteria to current antibiotics in use. Although many studies on these topics have been carried out, the data is subjected to quick changes as the spectrum of bacteria is different from one country to another and even between different geographical regions in the same country. With the wide usage of antibiotics in the past decades and the emergence of many new resistant drug resistant bacteria, it has become necessary to constantly evaluate bacteriology reports obtained from URTIs $[1,2,3]$. The purpose of this study was to investigate the current bacteriology profiles of complicated URTIs treated in our ENT Department, to uncover antibiotic susceptibility patterns and also bacterial resistance to antibiotics. The clinical importance of most antibiotic related research is to contribute to existing antibiotic guides, therefore finding out which type of bacteria causes infections more often in the key anatomical sites of the ENT sphere (nose/sinuses, middle-ear) was another goal of this study.

*email: razvan@ riaclinic.com, Phone: 0040727224447 REV.CHIM.(Bucharest) $\bullet 70 \diamond$ no. $12 \downarrow 2019$

The authors contributed equally to this article and should be considered first author 4426

http://www.revistadechimie.ro 


\section{Experimental part}

\section{Material and metods}

This prospective study was carried out on 82 consecutive patients admitted for complicated URTIs in an ENT Department, Craiova Emergency County Clinical Hospital , between January 2017 and January 2018. We considered bacterial infections that required hospitalization and/or surgical treatment as "complicated URTIs".

Bacteriology cultures were obtained differently depending on the site of the infection. Smears were prepared from the swab and stained by Gram stain. The swabs were cultured on a blood agar, MacConkey agar and chocolate agar plates. The plates were incubated overnight at $37^{\circ} \mathrm{C}$. Colony count was obtained from blood agar plate. The growth of the organism was characterized by colony morphology and Gram's staining from the culture plates. A detailed biochemical testing was performed \& antibiotic sensitivity testing was performed on Mueller-Hinton agar plates by Kirby-Bauer's disc diffusion method using suitable antibiotics as per CLSI (Central Laboratory Standard Institute) guidelines [3]. Aerobic and anaerobic cultures were tested for the collected specimen samples [4- 6]. Only cultures that revealed a moderate to heavy amount of bacterial growth were considered significant and included in this study. Fungi were excluded. Testing was also carried out for the Koch bacillus.

The antimicrobial agents tested were amikacin (AK), ampicillin (AM), amoxicillin/clavulanic acid (AMC), aztreonam (ATM),chloramphenicol (C),ceftazidime (CAZ), cefoperazone/sulbactam (CES), ciprofloxacin (CIP), clindamycin (CLI), clarithromycin (CLR), colistin (COL), cefpirome (CPO), ceftriaxone (CRO), cefotetan (CTT), cefotaxime (CTX), cefuroxime (CXM), cefazolin (CZ), doxycycline (DO), erythromycin (ERY), ertapenem (ETP), cefepime (FEP), cefoxitin (FOX),gentamicin (GEN),high-dose gentamicine (GENHL), imipenem/cilastatin (IMC), imipenem (IPM), levofloxacine (LEV), linezolid (LNZ), meropenem (MEM), moxifloxacin (MFX), myconazole (MIC), nitrofurantoin (NIT), norfloxacin (NOR), ofloxacin (OFX), oxacillin (OXA), penicillin (P), rifampicin (RIF), tetracycline (TE), teicoplanin (TEC), tigecycline (TGC), tobramycine (TOB), piperacillin / tazobactam (TPZ), vancomycin (VA).To assure the accuracy and reliability of antimicrobial susceptibility test, the reference strains S. aureus (ATCC25923), Escherichia coli (ATCC25922), and P. aeruginosa (ATCC 27853) were used as internal quality controls.

Statistical Analysis. The gathered data were checked for accuracy, entered and analysed using SPSS Version 20 Vatistical Voftware, Whonet 5.6 software (World Health Organisation, Geneva, Switzerland) and Microsoft Excel. Comparative analyses variables were made using the Student's t-test for two groups and one-way analysis of variance (ANOVA) for more than two groups. A value of $\mathrm{P}<0.05$ was considered to indicate a statistically significant difference.

\section{Results and discussions}

Acute or chronic bacterial pathology of the paranasal sinuses with complications that required surgical approach accounted for 19 cases out $(23.17 \%)$ of the total 82 cases admitted in the study. Acute or chronic middle ear disease, mastoiditis or complicated cholesteatoma that required surgical treatment accounted for 35 cases (42.68\%). Complicated suppurative tonsillitis in children and intratonsillar abscesses accounted for 10 cases (12.19\%). Parapharyngeal space abscesses accounted for only one case (1.219\%). Cellulitis of the head and neck accounted for 10 cases $(12.19 \%)$. Neoplasms of the pharynx and larynx with an acute ongoing bacterial superinfection accounted for 5 cases $(6.1 \%)$, and chronic diseases of the ears, nose, pharynx or larynx with suspicious or abnormal local discharge accounted for 2 cases $(2.43 \%)$.

Out of the total number of 82 patients, $52(63.4 \%)$ were male, and 30(36.6\%) were female. Male patients seem more likely to develop complications from bacterial URTIs, and complications are more likely to occur in adults than in children (ANOVA, $\mathrm{P}<0.001$ ).

Prevalence of Isolated Bacteria from Cultures. We analysed a total of 95 antibiograms, collected from 82 patients included in our study. A total number of $12(11.58 \%)$ tests came back negative. Out of all the positive results we were able to identify 14 different pathogenic bacteria species. Gram positive and negative bacteria were included. Among these bacterial species, the predominant bacterial isolates (over 50\% of the isolated strains) were Pseudomonas species (13 samples - 15.66\%) followed by Staphylococcus aureus MRSA (12 samples - 14,46\%) and non-MRSA (10 samples - 12.05\%) and Streptococcus species (11 samples - 13.25\%). Klebsiella species and Proteus species followed with 7 detected samples each (8.43\%). Streptococcus pneumoniae accounted for 6 samples (7.22\%). 5 samples $(6,02 \%)$ of Enterococcus species were detected. Escherichia coli and Gram Negative non-fermentative Bacilli accounted for 3 samples each (3.61\%). Coagulase-negative Staphylococci and Group A Beta-haemolytic Streptococcus accounted for 2 samples each (2.4\%). Haemophilus influenzae and Acinetobacter species were each detected in 1 sample $(1.2 \%)$.

Antibiotic Susceptibility and Resistance of Isolated Bacteria. We documented 43 strains of gram positive bacteria $(51.8 \%)$ and 40 strains of gram negative bacteria $(48.2 \%)$. We considered the bacteria to be sensitive to a certain antibiotic if over $90 \%$ of tested strains were susceptible to the tested drug. We noted moderate sensitivity if over $50 \%$ 
of tested strains were susceptible to the tested drug. We considered the bacteria to be moderately resistant if less than $50 \%$ of tested strains displayed susceptibility. Complete resistance to the drug was noted if $0 \%$ of tested strains were affected by the tested antibiotic. We used the same percentage indicator for the moderate sensitivity and moderate resistance of bacterial species as in the 'The European Database for antibiotic resistance. [7].

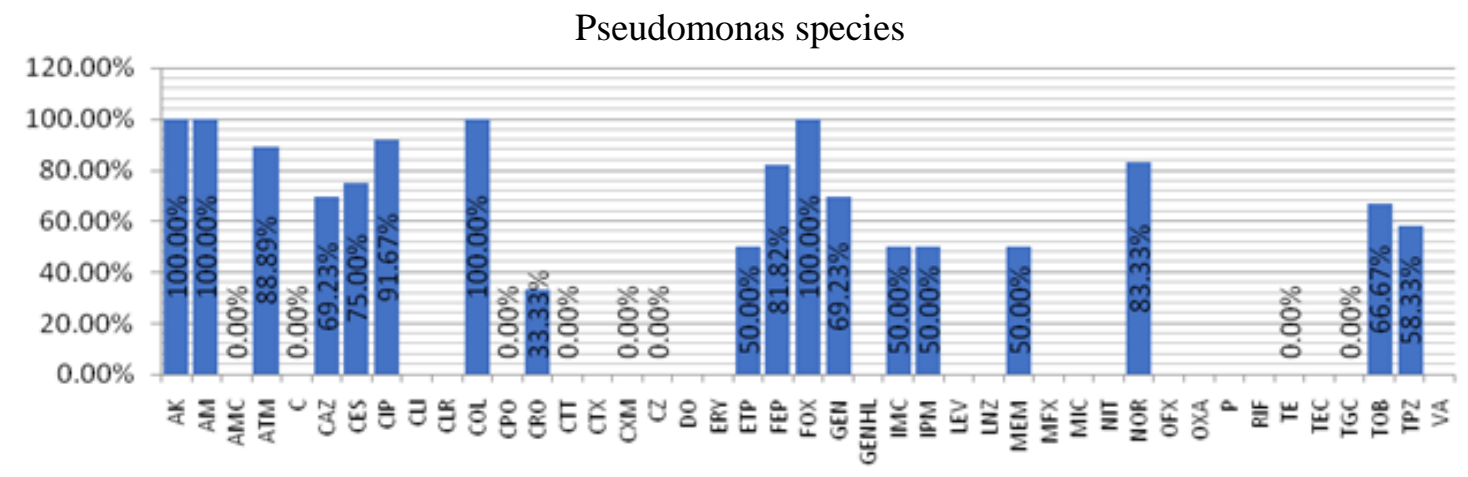

Chart 1. Antibiotic Susceptibility and Resistance for Pseudomonas sp.

The Pseudomonas species isolated were sensitive in over 90\% of tested strains to: AK, AM, CIP, COL, and FOX. Pseudomonas sp. were moderately sensitive (sensitive in over $50 \%$ of tested strains but under $90 \%$ ) to: ATM, CAZ, CES, FEP, GEN, NOR, TOB and TPZ. Pseudomonas was moderately resistant (50\% or more of tested strains resistant to the drug) to: CRO, ETP, IMC, IMP, MEM. The strains were completely resistant ( $0.00 \%$ susceptibility) to AMC, $\mathrm{C}, \mathrm{CPO}, \mathrm{CTT}, \mathrm{CXM}, \mathrm{CZ}, \mathrm{TE}$ and TGC.

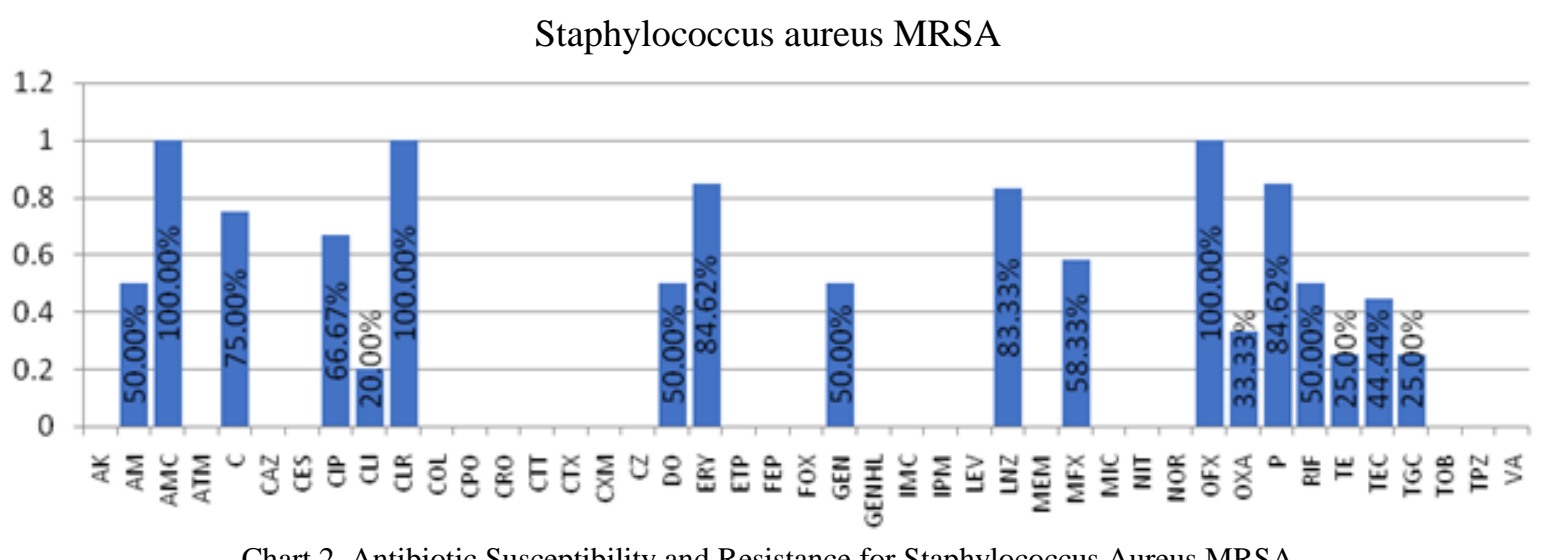

Chart 2. Antibiotic Susceptibility and Resistance for Staphylococcus Aureus MRSA

The isolated strains of Staphylococcus aureus MRSA were susceptible to the following antibiotics: AMC, CLR, OFX. The strains were moderately sensitive to: C, CIP, ERY, MFX, LNZ and P. We encountered moderate resistance resistance towards: AM, CLI, DO, GEN, OXA, RIF, TE, TEC and TGC.

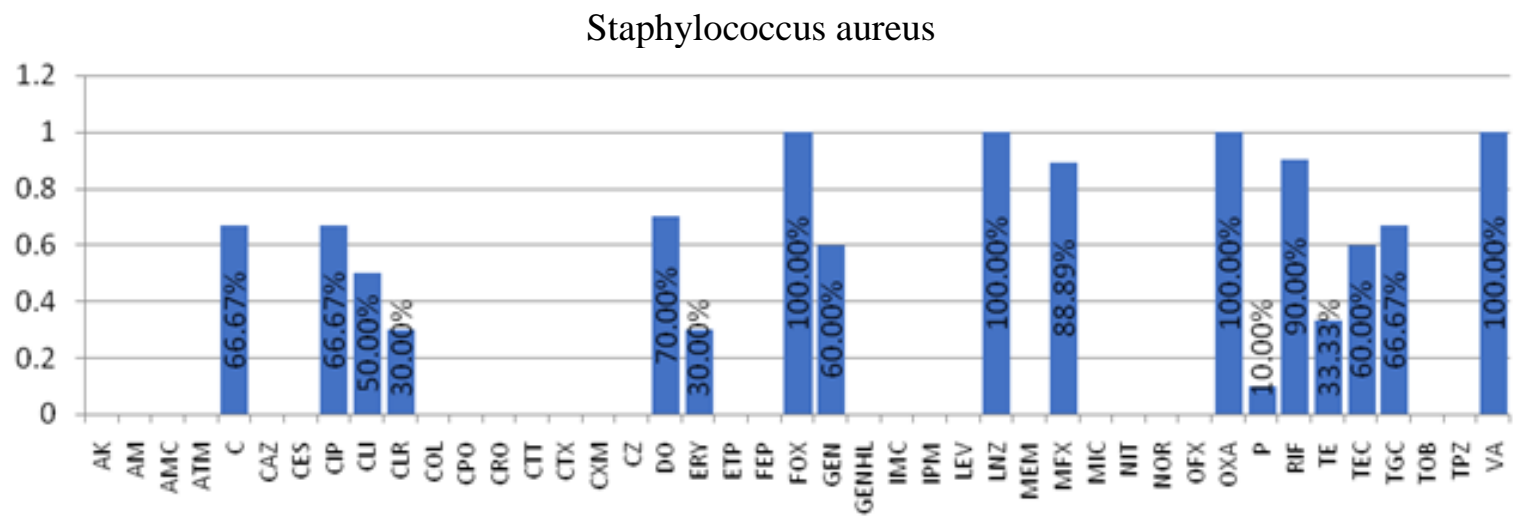

Chart 3. Antibiotic Susceptibility and Resistance for Staphylococcus Aureus non-MRSA

The isolated strains of Staphylococcus aureus non-MRSA were susceptible (sensitive in over $90 \%$ of tested strains) to the following antibiotics: FOX, LNZ, OXA, RIF and VA. The strains were moderately sensitive to: DO, MFX and RIF. We encountered moderate resistance (sensitive in less than $70 \%$ of tested strains) towards: C, CIP, CLI, CLR, 
ERY, GEN, P, TE, TEC and TGC.

The isolated strains of Klebsiella sp. were susceptible to the following antibiotics: C, CIP, COL, CPO, CTX, ETP, FEP, IMC, IPM, OFX and TGC. The strains were moderately sensitive to: ATM, CAZ and CRO, GEN and TPZ. We encountered moderate resistance towards: CXM, CZ and TOB. Klebsiella sp. were fully resistant ( $0 \%$ sensitivity) to: AM, AMC, FOX and TE.

The detected strains of Proteus sp. were sensitive to: AK, CES, and MFX. They were moderately sensitive to: C, CIP, CRO, FEP, IPM, GEN, TPZ and TOB. Moderate resistance was uncovered towards: AM, AMC, ATM, CAZ, COL, CXM, CZ, ETP, FOX and TGC. Complete resistance of the bacteria towards CPO, CTX, IMC and TE was detected.

The other isolated species of Streptococcus, non-beta-haemolytic, were abundant, accounting for 13,25\% of the total number of acute complicated bacterial infections. The isolated strains were sensitive to the following antibiotics: DO, FOX, GEN, IPM, LNZ, MEM, MFX, OFX, VA. Moderate sensitivity was registered towards: AM, C, CIP, CLI, CLR, CRO, CTX, P, TE and FEP. Streptococcus sp. was moderately resistant to:ERY, RIF, and TGC. No antibiotic susceptibility was detected for: OXA and TEC.

The most prevalent subspecie of Streptococcus was Streptococcus pneumoniae, detected in 6 cases of complicated bacterial infections $(6,31 \%)$. This determined us to analyse its antibiotic susceptibility separately from the species family. S. pneumoniae was sensitive in $100 \%$ of tested strains to: C, CIP, CLI, CRO, DO, ERY, FEP, GEN, IPM, LNZ, MEM, MFX, MIC, NOR, OXA, RIF and VA. Moderate sensitivity was encountered for TE. Moderate resistance towards AM, CTX and P was noted. Resistance to CLR was also noted.

The Enterococcus species isolated were sensitive to: FEP, GEN, GENHL, LNZ, P, TEC and VA. The strains were moderately sensitive to AM, DO and CIP. Moderated resistance was noted towards: ERY and NOR. Resistance was encountered for CLI, CLR, NIT and OXA.

For the rest of the bacteria encountered in our study we consider the data as being insufficient for further analysis, as each strain was encountered in 3 or less cultures. These bacteria species are: Escherichia coli, Gram Negative NonFermentative Bacilli, Group A Beta-hemolytic Streptococcus, Coagulase-negative Staphylococci, Haemophilus influenzae and Acinetobacter species.

It is now common knowledge for medical researchers that antibiotic resistance is a natural phenomenon caused by the common use as well as abuse of antibiotic therapy both outside and inside of hospitals. Although the emergence of new, drug-resistant bacteria is inevitable, the transformation of bacterial strains into resistant strains and further into drug-resistant bacterial populations is greatly favoured by non-rational and excessive antibiotic therapy.

In order to limit the non-rational usage of antibiotics, Antibiotic Guidelines are constantly being published and updated. The use of an Antibiotic Guideline, and standardized antibiotic treatment schemes for each bacterial infection seems to be the only logical way to deter the appearance of new drug-resistant bacterial populations. In order to maintain the effectiveness of a guideline, constant reanalysis of bacterial diseases, both active strains and antibiotic susceptibility, is a requirement. Bacterial diseases with notable complications despite proper antibiotic treatment should be studied for etiological bacterial strains. Antibiotic susceptibility and resistance should ideally be noted in each case. Bacterial genomics are deemed very important when bacterial drug resistance is being taken into account [8].

Our study focused on complicated bacterial diseases that we encountered during the 12 month period of monitoring patients in our ward. These cases were admitted in the hospital, most of them having taken previous courses of antibiotics, and were more likely to uncover new changes in how the bacterial populations react to antibiotics. Gram positive and gram negative bacteria were both included in the study, and all statistically relevant strains were analysed. For the ENT spectrum of URTIs, we uncovered that more than 50\% of diseases were caused by 4 pathogens: Pseudomonas species, Staphylococcus aureus (non-MRSA and MRSA included) and Streptococcus species (excluding beta-hemolytic group A streptococci and Streptococcus pneumoniae). The percentage of gram positive and gram negative bacteria did not show a statistically significant difference in favour of either germ populations. The complicated bacterial diseases we encountered during our study mostly involved the middle-ear or the paranasal sinuses. More than $60 \%$ of our study group patients had a bacterial infection involving one of those anatomical regions. The most frequent bacterial diseases in the ENT sphere that developed complications were acute and chronic otitis and mastoiditis.

Most complicated bacterial infections involving the pharynx (complicated suppurative tonsillitis in children, intratonsillar abscess, parapharyngeal space abscess) were caused by Streptococcus species (the most prevalent germ), Staphylococcus aureus non-MRSA and Enterococcus. The disease with the highest life-threatening risk in this group was the parapharyngeal space abscess, for which Enterococcus faecalis was responsible.

The complicated cases of cellulitis of the neck, face or involving cervical ganglia, were mainly caused by Staphylococcus aureus MRSA infections, followed by the non-MRSA Staphylococcus.

The acute and chronic bacterial pathology of the nose and paranasal sinuses that required immediate surgical approach, were mainly caused by germs as Streptococcus species and Staphylococcus aureus non-MRSA. However, 
the third most identified germ in this diseases' group was the Staphylococcus aureus MRSA. The most common complication for this group of diseases was orbital cellulitis.

The middle-ear and mastoid bone pathology that required surgical treatment was caused by a plethora of germs, as we managed to isolate Pseudomonas species, Proteus species, MRSA and non-MRSA Staphylococcus species, Streptococcus pneumoniae, gram negative non-fermentative bacilli as well as Klebsiella species. The most prevalent of the germs isolated was however Pseudomonas species, followed by Proteus and MRSA Staphyloccous and Klebisella. The most frequent complications that arose in this disease group were: meningitis, lateral sinus thrombophlebitis, and cerebral abscess.

In the case of Pseudomonas, known as a bacteria that easily colonizes hospital wards and medical equipment, the testing revealed curious results with susceptibility in over $90 \%$ of tested strains to amikacyn (AK), ampicillin (AM), ciprofloxacin (CIP), colistin (COL), and cefoxitin (FOX), and resistance to amoxicillin/clavulanic acid (AMC), chloramphenicol $(\mathrm{C})$, cefpirome $(\mathrm{CPO})$, cefotetan $(\mathrm{CTT})$, cefuroxime $(\mathrm{CXM})$, cefazolin $(\mathrm{CZ})$, tetracycline (TE), (tigecycline) TGC. We noted good susceptibility to ampicillin and cefoxitin, and resistance to second-generation cephalosporins. We attribute the good susceptibility to cefoxitin due to the cephamycins' structure based upon the cephem nucleus, granting efficiency towards anaerobic microbes. The curious spectrum of resistance and susceptibility may also be caused by different species of drug-resistant Pseudomonas and less resistant species, depending on their prior exposure to the hospital environment and to antibiotic treatment.

The isolation of Staphylococcus aureus MRSA from surgical wounds or cavities in the human body has been shown to be related to lower chances of primary healing due to complications after surgery [9]. The current method for testing MRSA is the resistance test to cefoxitin [10], which is also a surrogate test for resistance to oxacillin. The MRSA Staphylococcus may also colonize hospital wards and become difficult to control or eradicate. Its survival is promoted probably by the high usage of over the counter antibiotics, that can induce the transformation of methicillin susceptible Staphylococcus aureus (MSSA) in MRSA [11]. In our study, the prevalence of Staphyolococcus MRSA was $14.46 \%$.

Analyzing further, the problem is more complicated, as many patients are already colonized at admission with nonMRSA strains (MSSA included), mostly located in the pharynx [12]. Studies even show patients with acne, from which Staphylococcus aureus has been isolated [13]. Some of these patients may have already undergone abusive selfimposed antibiotic treatments, or even non-rational treatments prescribed by their general practitioner. These MRSA subpopulations can be selected through exposure to cefoxitin or oxacillin in up to 48 hours [11]. It is therefore difficult to place the moment in which a non-MRSA strain may convert into a MRSA strain, and even if that moment takes place inside or outside of the hospital.

As expected, the antibiotic susceptibility of both Staphylococcus MRSA and non-MRSA strains is highly different, with only 3 antibiotics accounting for sensitivities over 60\% (moderately sensitive) in both types of bacterial populations: chloramphenicol (C), ciprofloxacin (CIP) and linezolid (LNZ). The expected low resistance to rifampicin (RIF), that most studies report, was not well-grounded with our findings in the ENT sphere, as only 50\% of the Staphylococcus MRSA strains were sensitive.

The other most encountered type of bacteria in our study was the Streptococcus species. We classified the types of Streptococcus bacteria by species. Contrary to older data in literature, we came across little Beta-haemolytic Group A Streptococcus (2 samples accounting for $2.4 \%$ of all documented cultures), which we found to be of no statistical importance to our study. Streptococcus pneumoniae accounted for 6 samples $(7,22 \%)$, and the most abundant types of Streptococcus were other species like S. viridans and S. pyogenes. Antibiotic susceptibility testing was carried out separately for the Streptococcus species (S. viridans and S. pyogenes) which were mainly identified in the pharynx and larynx, and separately for Streptococcus pneumoniae, which was identified in middle ear culture samples. Streptococcus pneumoniae was sensitive to most antibiotics tested, with moderate resistance to (CTX) and (P), and complete resistance to clarithromycin (CLR). The Streptococcus species (S. viridans and S. pyogenes) identified in the pharynx and sometimes in the larynx, displayed different patterns of susceptibility. Overall we may conclude that the Streptococcus species inhabiting the pharynx and/or larynx are more resistant to drugs, whereas S. pneumoniae which is mostly detected in samples collected from patients with otitis and mastoiditis, is still a highly antibiotic-sensitive bacteria. This difference may arise from irrational self-medication with antibiotics for common colds (a practice still common in Romania) or from the fact that most of acute tonsillitis or pharyngitis cases diagnosed by general practitioners rarely end up in being put on the appropriate course of antibiotics $[14,15]$.

\section{Conclusions}

Bacterial strains that cause infections with a high risk of complications in the otorhinolaryngological spectrum are certainly different between hospitals and regions alike..

Some bacterial strains isolated from our hospitalized patients have a higher resistance to certain antimicrobials, like higher-generation cephalosporins, linezolid or carbapenems, making the clinician doctor face a terrible dilemma when starting antibiotic therapy in unclear cases. 


\section{References}

1. VAN BELKUM A, BACHMANN TT, et al.. Nature Reviews. Microbiology. 17, nr 1, 2019, p. 51-62.

2. THOMAS JK, FORREST A, BHAVNANI SM, et al. Antimicrobial Agents and Chemotherapy. 42, nr. 3, 1998, p. $521-7$.

3. KASSIM A, OMUSE G, PREMJI Z, REVATHI G. Ann Clin Microbiol Antimicrob 15, nr. 21, 2016.

4. GEBRE-SEALSSSIE S. Ethiopian Medical Journal, 45, nr. 4, 2007, p. 363-370.

5. LI JY, MA Y, SUN Z, YAO L, ZHANG L, HU C AND JIN S: Cluster analysis to detect the homogeneous strains of E. coli isolated from clinical specimens. Chin J Microbiol Immunol 23: 384-387, 2003.

6. XU J, SHI C, SONG M, et al.J Food Sci 79, 2014, p. M635-M642.

7. Data from the ECDC Surveillance Atlas - Antimicrobial resistance. http://www.ecdc.europa.eu/en/healthtopics/ antimicrobial_resistance/ database/Pages/database.aspx.

8. CĂLINA D, DOCEA AO, ROSU L, et al. Mol Med Rep., 15, nr. 2, 2017, p. 681-688.

9. SOLOMKIN JS. Crit. Care. Med. 29, Suppl 4, 2001, p. N97-N 99.

10. COCKERILL FR, PATEL JB. Clin Lab Stand Inst 35, 2015, p. 44-49.

11. UNGUREANU A, ZLATIAN O, MITROI G, et al. Molecular Medicine Reports, 16, 2017, p. 8771-8780.

12. OWENS CD, STOESSEL K. J Hosp Infect 70, Suppl 2, 2008, p. 3-10.

13. THORNSBERRY C, MCDOUGAL LK. J Clin Microbiol, 18, 1983, p. 1084-1091.

14. DZIDIC S, BEDEKOVIĆ V. Acta Pharmacol Sin, 24, 2003, p. 519-526.

15. PETERSON LR, QUICK JN, JENSEN B, et al : Arch Intern Med, 150, 1990, p. 2151-2155.

Manuscript received: 23.10 .2019 